\title{
Comparison of double-folding effective interactions within the cluster model
}

\author{
B. D. C. Kimene Kaya* and S. M. Wyngaardt \\ Department of Physics, University of Stellenbosch, P.O. Box 1529, Stellenbosch 7599, South Africa \\ T. T. Ibrahim \\ Department of Physics, Federal University Lokoja, PMB 1154, Lokoja, Nigeria \\ W. A. Yahya \\ Department of Physics and Materials, Kwara State University, PMB 1530, Malete, Nigeria
}

(Received 11 June 2018; published 10 October 2018)

\begin{abstract}
Cluster-core hybrid potentials with Woods-Saxon plus cubic terms have been constructed to account for both the decay properties and positive-parity ground-state bands in ${ }^{212} \mathrm{Po},{ }^{218} \mathrm{Rn},{ }^{222} \mathrm{Ra}$, and ${ }^{228} \mathrm{Th}$. The hybrid potential parameters have been extracted from the real part of the double-folding interaction using the realistic Michigan3-Yukawa (M3Y) and a complex Gaussian effective interactions. We find that both the effective interactions exhibit similar behavior in the internal region, and the agreement between our estimated results with the existing experimental data is satisfactory.
\end{abstract}

DOI: 10.1103/PhysRevC.98.044308

\section{INTRODUCTION}

The existence of clustering effects in nuclei has been proven from experimental and theoretical points of view in both light and heavy nuclear regions. These include $\alpha$ and heavy cluster radioactivity, $\alpha$ transfer reactions, enhanced electromagnetic transitions, and more recently the interpretation of the $7.65 \mathrm{MeV}{ }^{12} \mathrm{C}$ Hoyle state [1-13]. Several microscopic approaches such as the resonating group method (RGM), the generator coordinate method, and the orthogonality condition method (OCM) have been used extensively to study the structure of light nuclei $[14,15]$. Here we consider a simplified form of the RGM which provides an intuitive approach for understanding the nuclear structure. It describes a nucleus as being formed from an inert core of nucleons and a cluster of strongly correlated nucleons orbiting the core. The model holds provided that the nuclei involved present a most stable configuration against any internal breakup [16]. For heavy nuclei, it may be viewed as lying in the interplay between their deformation and decay properties. Consequently the many-body problem is reduced to a two-body solvable problem with an appropriate cluster-core interaction. Earlier studies have shown that the eigenstates of the cluster-core local potential generate bands of cluster states with structure properties comparable to experimental findings [11,12,16-18]. The form of the two-body interaction required for describing the observed properties in a unified scheme is therefore important. Recently, we proposed a hybrid interaction constructed from both the Saxon-Woods and M3Y interactions which satisfactorily reproduced the observed experimental data [17].

\footnotetext{
*kimenekaya@sun.ac.za, kaya.christel@gmail.com
}

In this study, we compare the predictive power of the hybrid interactions generated from different nucleon-nucleon $(N N)$ effective interactions. In particular we compare the calculated energy spectra and the decay properties of representative nuclei using hybrid potential parameters generated from the M3Y and the successful complex Gaussian effective interactions. This paper is organized as follows. In Sec. II, we discuss the cluster-core configuration. The theoretical framework is presented in Sec. III. The discussion of our results is presented in Sec. IV, and the conclusion is given in Sec. V.

\section{CONFIGURATION OF CLUSTER-CORE MODEL}

In this study our choices of the parent nuclei and the cluster-core configuration are based on the following criteria: First, the parent nuclei have been observed to radioactively decay, except ${ }^{218} \mathrm{Rn}$, leaving a fixed ${ }^{208} \mathrm{~Pb}$ core. Second, the cluster charge $Z$ and neutrons $N$ numbers are found to obey a regular mathematical series

$$
Z_{j}=j Z_{0}, \quad Z_{0}=2,
$$

and

$$
N_{j}=N_{j-1}+r, \quad N_{0}=0,
$$

where

$$
r= \begin{cases}2 & \text { if } j=1,3,5, \ldots \\ 4 & \text { if } j=2,4,6, \ldots\end{cases}
$$

for $j=1,2,3,4$, giving rise to the configuration ${ }^{208} \mathrm{~Pb}+$ ${ }_{Z_{j}}^{A_{j}} \mathrm{X}_{N_{j}}$. We see that the constituent nucleons of the clusters are lumped together outside the doubly-magic core ${ }^{208} \mathrm{~Pb}$. Thus the ground-state configuration of the parent nuclei may then 
be written in a compact notation

$$
\left[\pi\left(h_{9 / 2}\right)^{Z_{j}} v\left(g_{9 / 2} i_{11 / 2}\right)^{N_{j}}\right]_{0^{+}},
$$

where $\pi$ and $v$ represent the protons and neutrons such that $Z_{j} \leqslant 8$ and $N_{j} \leqslant 12$. Therefore, the cluster model assumes a preformed cluster inside the parent nucleus moving within the mean-field potential created by the remaining core nucleons.

Many works have been devoted to investigating possible core-cluster partitions for an arbitrary parent nuclei. Notable are the methods based on the binding-energy systematics formulated to determine the possible core-cluster partition for heavy nuclei. These works may be considered to serve as a test to benchmark the predicted cluster configurations in regions such as the rare-earth and superheavy nuclei [18-21].

\section{THEORY}

\section{A. Energy spectra}

The two-body relative motion generating the cluster states is appropriately described by the Schrödinger wave equation

$$
\left(-\frac{\hbar^{2}}{2 \mu} \frac{d^{2}}{d r^{2}}+V(r)\right)|n, l, m\rangle=E_{n l}|n, l, m\rangle,
$$

where

$$
|n, l, m\rangle=\frac{\varphi_{n l}(r)}{r} Y_{l m}(\hat{r})
$$

is the state wave function with energy $E_{n l}, \mu$ is the reduced mass, and $V(r)$ is the total interaction between the core and the cluster. The quantities $n, l$, and $m$ are respectively the principal, orbital, and azimuthal quantum numbers, and the symbol $\hat{r}=(\theta, \phi)$ denotes the angular coordinates.

The level structure characterizing the collective fluctuations around the equilibrium state of the core may straightforwardly be determined from the Bohr-Sommerfeld quantization rule

$$
\int_{r_{1}}^{r_{2}} \sqrt{\frac{2 \mu}{\hbar^{2}}\left[E_{n l}-V(r)\right]} d r=(G-l+1) \frac{\pi}{2} .
$$

Here $G=2 n+l$ is the total global quantum number [11], whose value is chosen such that the energetically favored nucleon correlations are located just above the Fermi surface of the core. The energies $E_{n l}=Q+E_{l}^{*}$, where $E_{l}^{*}$ are the excitation energies for the states $l^{\pi}=0^{+}, 2^{+}, 4^{+}, \ldots$, characterizing the low-lying positive-parity ground-state band. The quantity $Q$ corresponds to the ground state $Q$ value corrected for electron shielding [22].

The electromagnetic transitions between the cluster states are described by "single-particle" transitions of a special kind involving all the cluster nucleons instead of a single proton or neutron. Since the cluster and the core are considered to be spherical in their ground state, the matrix elements of their intrinsic quadrupole moment is zero. The relative motion electric quadrupole operator is then given by [23]

$$
\mathcal{M}_{20}=\beta_{2} r^{2} Y_{20}(\hat{r}),
$$

from which we can deduce the in-band transition nondiagonal matrix elements

$$
B(E 2)=\frac{2 l_{f}+1}{2 l_{i}+1}\left|\left\langle n_{f}, l_{f} \| \beta_{2} r^{2} Y_{20}(\hat{r})|| n_{i}, l_{i}\right\rangle\right|^{2},
$$

where $\left(n_{f}, l_{f}\right)$ and $\left(n_{i}, l_{i}\right)$ are the principal and orbital quantum numbers for the final and initial nuclear states. The charge-dependent factor $\beta_{2}$ defining the recoil term is given by

$$
\beta_{2}=\frac{Z_{1} A_{2}^{2}+Z_{2} A_{1}^{2}}{\left(A_{1}+A_{2}\right)^{2}}
$$

\section{B. Root-mean-square radii}

The mean-square charge radius of the parent ground state is related to the rms charge radii of the cluster and daughter nuclei as follows [23]:

$$
\left\langle r_{\mathrm{ch}}^{2}\right\rangle=\frac{Z_{1}}{Z}\left\langle r_{\mathrm{ch} 1}^{2}\right\rangle+\frac{Z_{2}}{Z}\left\langle r_{\mathrm{ch} 2}^{2}\right\rangle+\frac{Z_{1} A_{2}^{2}+Z_{2} A_{1}^{2}}{Z A^{2}}\left\langle r_{0}^{2}\right\rangle,
$$

where $\left\langle r_{\mathrm{ch} 1}^{2}\right\rangle$ and $\left\langle r_{\mathrm{ch} 2}^{2}\right\rangle$ are the core and cluster mean-square charge radii with values taken from experimental data. The quantity $\left\langle r_{0}^{2}\right\rangle$ is the mean-square separation of the core-cluster system defined as

$$
\left\langle r_{0}^{2}\right\rangle=\left\langle n, l\left|r^{2}\right| n, l\right\rangle=\int_{0}^{\infty} r^{2} \varphi_{n l}^{2}(r) d r .
$$

This observable is directly related to the proton density distribution and thus provides a good test of the relative-motion wave function in addition to the transition moments described above. We shall see how this quantity varies for a fixed core when the cluster charge and neutron numbers increase.

\section{Static quadrupole moment}

The static quadrupole moment, which is a measure of the deviation of nuclear charge distribution from spherical symmetry, provides also an opportunity for detailed tests of cluster-state wave functions. Thus, the collectivity of moving particles (cluster constituents) around an inert core can be directly probed using the static quadrupole moments [24]. For the nuclear state of an axially deformed nucleus with angular momentum $l$ (and $m=l$ ), the moment is given by

$$
\begin{aligned}
Q_{2} & =\sqrt{\frac{16 \pi}{5}}\left\langle n, l, m\left|\mathcal{M}_{20}\right| n, l, m\right\rangle \\
& =-\beta_{2} \frac{2 l}{2 l+3} \int_{0}^{\infty} r^{2} \varphi_{n l}^{2}(r) d r .
\end{aligned}
$$

\section{Decay half-life}

The clearest evidence of clustering is seen through the decay of parent nuclei. The cluster decay half-life, which is the observable of interest, is defined as

$$
T_{1 / 2}=\frac{1}{P} \frac{2 \mu \ln 2}{\hbar} \exp \left[2 \int_{r_{2}}^{r_{3}} k(r) d r\right] \int_{r_{1}}^{r_{2}} \frac{d r}{k(r)},
$$

where $r_{1}, r_{2}$, and $r_{3}$ are the turning points in order of increasing values and determined by numerically solving the 
equation $V(r)=E_{n l}$. The factor $P$ is the probability of cluster-core preformation and the wave number $k(r)$ is given by

$$
k(r)=\sqrt{\frac{2 \mu}{\hbar^{2}}\left|E_{n l}-V(r)\right|} .
$$

\section{E. Potential}

The total interaction between the core and the cluster, for a spherically symmetric system, is given by the sum of the nuclear $U_{N}(r)$, the Coulomb $U_{C}(r)$, and the rotational $U_{l}(r)$ potentials

$$
V(r)=U_{N}(r)+U_{l}(r)+U_{C}(r),
$$

where the Coulomb interaction is simply given by

$$
\begin{aligned}
U_{C}(r) & =\frac{Z_{1} Z_{2} e^{2}}{r}, \quad \text { if } r \geqslant R_{c}, \\
& =\frac{Z_{1} Z_{2} e^{2}}{2 R_{c}}\left(3-\left|\frac{r}{R_{c}}\right|^{2}\right), \quad \text { if } r \leqslant R_{c},
\end{aligned}
$$

with the Coulomb radius $R_{C}$ taken as the nuclear radius $R$ and the centrifugal component $U_{l}(r)$ is given by

$$
U_{l}(r)=\frac{l(l+1) \hbar^{2}}{2 \mu r^{2}}
$$

The assumption of a spherical system for a doubly-magic core ${ }^{208} \mathrm{~Pb}$ plus light cluster masses ${ }^{4} \mathrm{He}$ and ${ }^{10} \mathrm{Be}$ is found to be a good approximation in the decay and the spectroscopic calculations involving the total potential given in Eq. (15). On the other hand, for larger cluster sizes such as ${ }^{14} \mathrm{C}$ and ${ }^{20} \mathrm{O}$ the parent nuclei are deformed emitters with the deformation effect playing an important role in their decay half-lives [25].
TABLE I. Coefficients $G_{D_{i}}(\mathrm{MeV}), G_{E_{i}}(\mathrm{MeV})$, and $r_{v_{i}}\left(\mathrm{fm}^{-2}\right)$ for the CEG83.

\begin{tabular}{llllllllll}
\hline \hline$G_{D_{1}}$ & $G_{D_{2}}$ & $G_{D_{3}}$ & $G_{E_{1}}$ & $G_{E_{2}}$ & $G_{E_{3}}$ & $r_{v_{1}}$ & $r_{v_{2}}$ & $r_{v_{3}}$ \\
\hline 954.15 & -185.21 & -0.685 & 165.86 & -105.04 & -2.35 & -4.0 & -1.26 & -0.16 \\
\hline \hline
\end{tabular}

This necessitates using deformed Coulomb and nuclear interactions. For these latter parent nuclei, however, the groundstate deformation parameters are of the order of $\sim 0.1$ allowing the approximate treatment as spherical systems since the effect is more pronounced for larger deformation.

For the nuclear potential, we thus adopt the phenomenological Saxon-Woods plus Saxon-Woods cubed (SW+SW3)

$$
U_{N}(r)=-V_{0}\left[\frac{x}{1+\exp \left(\frac{r-R}{a}\right)}+\frac{1-x}{\left[1+\exp \left(\frac{r-R}{3 a}\right)\right]^{3}}\right],
$$

which has been applied with good success to obtain the ground-state band and surface properties of light and heavy nuclei. The function depends on the depth $V_{0}$, the mixing parameter $x$, the radius $R$, and the diffuseness $a$, that are usually optimized to fit experimental data. However, it provides little information on the microscopic nature of the clustering effects, even for the optimally closed-shell nuclei. A more microscopic approach involves the use of a doublefolding model (DFM) with realistic M3Y-NN interaction for the cluster-core system [8,26-29]. This potential is able to give a good account of elastic scattering properties as well as ground-state decay half-lives of $\alpha$-conjugate nuclei, but fails to predict consistent results for heavy-ion emission in the actinide region. In addition, the predicted level structures
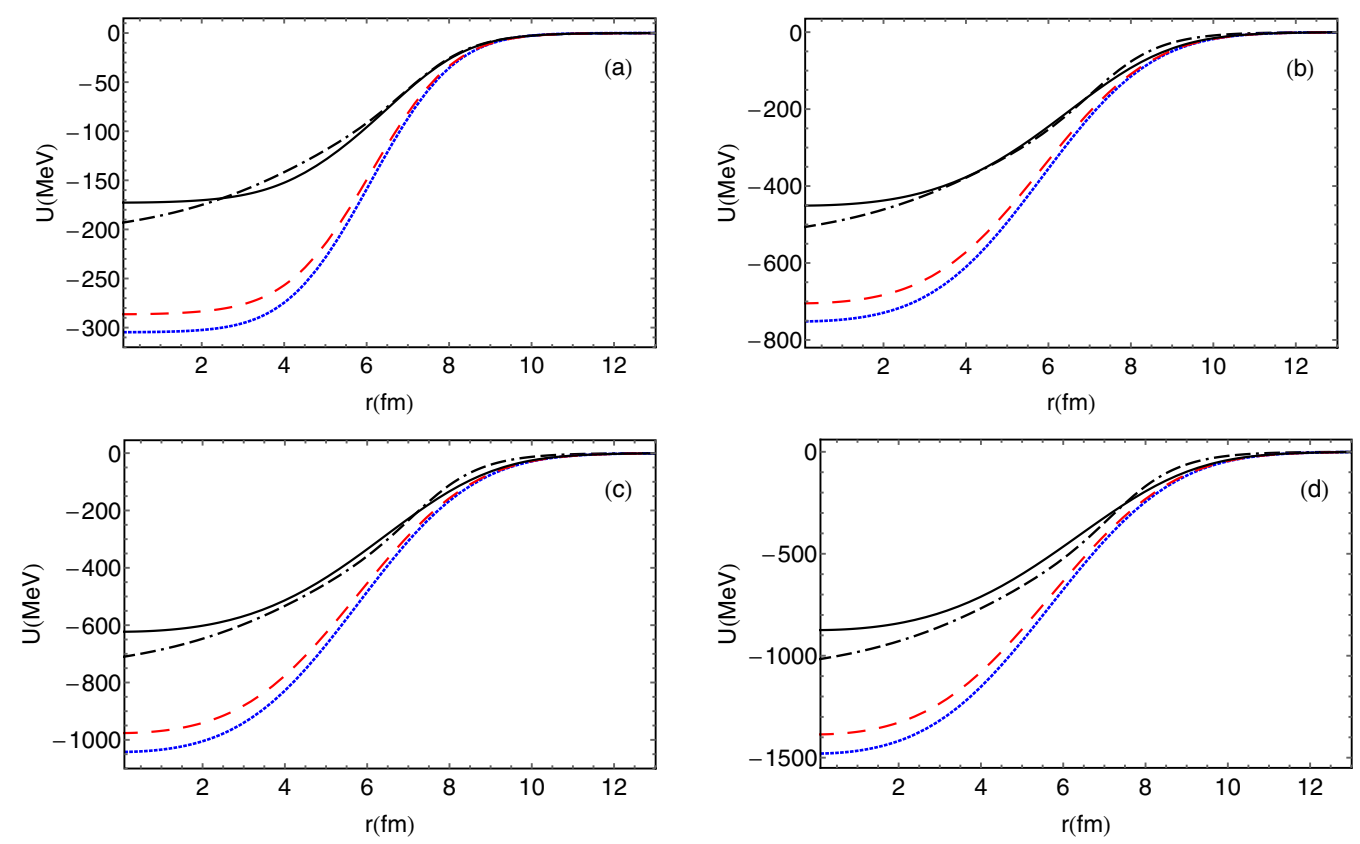

FIG. 1. The nuclear interactions for the ${ }^{208} \mathrm{~Pb}$ core plus (a) ${ }^{4} \mathrm{He}$ cluster, (b) ${ }^{10} \mathrm{Be}$ cluster, (c) ${ }^{14} \mathrm{C}$ cluster, and (d) ${ }^{20} \mathrm{O}$ cluster. The M3Y (dotted blue line) and CEG83a (dashed red line) are supplemented with zero-range exchange interactions. The CEG83b with finite-range exchange interaction and the phenomenological Saxon-Woods potential SW3 are represented with black solid and dot-dashed lines respectively. 
TABLE II. Normalization constant for different nuclear potentials.

\begin{tabular}{lcccc}
\hline \hline Cluster-core & $\lambda_{\text {M3Y }}$ & $\lambda_{\text {CEG83a }}$ & $\lambda_{\text {CEG83b }}$ & $G$ \\
\hline${ }_{2}^{4} \mathrm{He}+{ }_{82}^{208} \mathrm{~Pb}$ & 0.53 & 0.56 & 0.90 & 18 \\
${ }_{4}^{10} \mathrm{Be}+{ }_{82}^{208} \mathrm{~Pb}$ & 0.54 & 0.57 & 0.82 & 50 \\
${ }_{6}^{14} \mathrm{C}+{ }_{82}^{208} \mathrm{~Pb}$ & 0.53 & 0.56 & 0.80 & 70 \\
${ }_{8}^{20} \mathrm{O}+{ }_{82}^{208} \mathrm{~Pb}$ & 0.52 & 0.56 & 0.80 & 100 \\
\hline \hline
\end{tabular}

are either inverted for ${ }^{212}$ Po or compressed for heavy nuclei treated as core plus an exotic cluster. The success and the drawbacks of this interaction may be explained by the surface character of the clustering effects. This is related to the feature of the potential where the nuclear density falls to its minimal values resulting in a very large width. The spectroscopic calculations are also found to depend on the shape of the potential in the internal region. Following Refs. [17,28,29] we construct the hybrid potential which takes the form of Eq. (18) with parameters $V_{0}, a, R$, and $x$ determined from the surface part of the DFM using both the M3Y and complex Gaussian effective $N N$ interactions [30]. We note that the complex Gaussian effective interaction (CEG) is implicitly density dependent and has been found to reproduce consistently the equilibrium density and the binding energy of normal nuclear matter [31].

In the lowest approximation, the DFM potential is expressed as [32]

$$
U_{N}(\boldsymbol{r})=\lambda\left[U_{D}(\boldsymbol{r})+U_{\mathrm{EX}}(\boldsymbol{r})\right],
$$

with $\lambda$ being the normalization constant. The direct part is written as

$$
U_{D}(\boldsymbol{r})=\iint \rho_{A_{1}}\left(\boldsymbol{r}_{1}\right) \rho_{A_{2}}\left(\boldsymbol{r}_{2}^{\prime}\right) V_{D}(\boldsymbol{s}) d \boldsymbol{r}_{1} d \boldsymbol{r}_{2}^{\prime}
$$

where the effective $N N$ interaction $V_{D}(s)$ for the M3Y is given by

$$
V_{D}(s)=7999 \frac{\exp (-4 s)}{4 s}-2134 \frac{\exp (-2.5 s)}{2.5 s} .
$$

The exchange component $U_{\mathrm{EX}}(\boldsymbol{r})$, which in principle is nonlocal, may be approximated with the local form

$$
\begin{aligned}
U_{\mathrm{EX}}(\boldsymbol{r})= & \iint \rho_{A_{1}}\left(\boldsymbol{r}_{1}, \boldsymbol{r}_{1}+\boldsymbol{s}\right) \rho_{A_{2}}\left(\boldsymbol{r}_{2}^{\prime}, \boldsymbol{r}_{2}^{\prime}-\boldsymbol{s}\right) \\
& \times V_{\mathrm{EX}}(\boldsymbol{s}) \exp \left[\frac{i k(r) s}{\mu}\right] d \boldsymbol{r}_{1} d \boldsymbol{r}_{2}^{\prime},
\end{aligned}
$$

TABLE III. Optimized parameters for SW3 potential from M3Y.

\begin{tabular}{lcccc}
\hline \hline Cluster-core & $V_{0}(\mathrm{MeV})$ & $a(\mathrm{fm})$ & $x$ & $R(\mathrm{fm})$ \\
\hline${ }_{2}^{4} \mathrm{He}+{ }_{82}^{208} \mathrm{~Pb}$ & 206.904 & 0.725 & 0.330 & 6.807 \\
${ }_{4}^{10} \mathrm{Be}+{ }_{82}^{208} \mathrm{~Pb}$ & 506.810 & 0.977 & 0.510 & 6.715 \\
${ }_{6}^{14} \mathrm{C}+{ }_{82}^{208} \mathrm{~Pb}$ & 704.995 & 1.015 & 0.490 & 6.736 \\
${ }_{8}^{20} \mathrm{O}+{ }_{82}^{208} \mathrm{~Pb}$ & 1003.460 & 1.042 & 0.460 & 6.797 \\
\hline \hline
\end{tabular}

TABLE IV. Optimized parameters for SW3 potential from CEG83a.

\begin{tabular}{lcccc}
\hline \hline Cluster-core & $V_{0}(\mathrm{MeV})$ & $a(\mathrm{fm})$ & $x$ & $R(\mathrm{fm})$ \\
\hline${ }_{2}^{4} \mathrm{He}+{ }_{82}^{208} \mathrm{~Pb}$ & 210.600 & 0.750 & 0.350 & 6.726 \\
${ }_{4}^{10} \mathrm{Be}+{ }_{82}^{208} \mathrm{~Pb}$ & 505.310 & 0.990 & 0.520 & 6.700 \\
${ }_{6}^{14} \mathrm{C}+{ }_{82}^{208} \mathrm{~Pb}$ & 704.463 & 1.031 & 0.500 & 6.712 \\
${ }_{8}^{20} \mathrm{O}+{ }_{82}^{208} \mathrm{~Pb}$ & 1000.936 & 1.057 & 0.470 & 6.778 \\
\hline \hline
\end{tabular}

where the momentum is

$$
k^{2}(r)=\frac{2 \mu}{\hbar^{2}}\left[E_{c}-U_{N}(r)-U_{C}(r)\right]
$$

for the finite-range nucleon-nucleon interaction [32]. The quantity $\mu=A_{1} A_{2} M /\left(A_{1}+A_{2}\right)$ is the reduced mass, $M$ represents the nucleon mass, and $E_{c}$ the relative energy in the center of mass which is actually the decay $Q$ value. We emphasize here that the reduced mass $\mu$ may appropriately be taken as the nuclear inertia requiring the use of the effective mass parameter or mass tensor associated with the decay process at the separation distance between the centers of mass of the two fragments [33]. In principle, this may be calculated from using the Werner-Wheeler approximation [34] or from a more microscopic cranking model [35], if the dynamics of the dinuclear system is considered as uniaxial rotational motion of a quasi-particle quantum fluid with the rigid-flow kinematic moment of inertia $\mathfrak{I}=\mu r^{2}$ [36]. To a good approximation, we use the reduced mass $\mu$ since the effective nuclear inertia of a binary system at the touching point equals the reduced mass [35]. Here $U_{N}(r)=U_{D}(r)+U_{\mathrm{EX}}(r)$ and $U_{C}(r)$ represent the total nuclear and Coulomb potentials, respectively. It may alternatively be approximated with a pseudointeraction

$$
U_{\mathrm{EX}}(\boldsymbol{r})=\iint \rho_{A_{1}}\left(\boldsymbol{r}_{1}\right) \rho_{A_{2}}\left(\boldsymbol{r}_{2}^{\prime}\right) V_{\mathrm{EX}}(\boldsymbol{s}) \delta(s) d \boldsymbol{r}_{1} d \boldsymbol{r}_{2}^{\prime}
$$

in the zero-range limit with effective exchange term

$$
V_{\mathrm{EX}}(s)=-276\left(1-0.005 \frac{E_{c}}{A_{2}}\right) \delta(s) .
$$

Here $s=\boldsymbol{r}+\boldsymbol{r}_{2}^{\prime}-\boldsymbol{r}_{1}$ is the relative coordinate between a nucleon at the spatial position $\boldsymbol{r}_{1}$ with respect to the center of mass (c.m.) of nucleus $A_{1}$ and another nucleon at the spatial position $\boldsymbol{r}_{2}^{\prime}$ with respect to the c.m. of nucleus $A_{2}$, and $\boldsymbol{r}$ is the relative coordinate between the centers of mass of the interacting nuclei. Here the ground-state density distribution is taken as a Gaussian form [37],

$$
\rho_{A_{2}}(r)=0.4299 \exp \left(-0.702 r^{2}\right),
$$

for an $\alpha$ cluster, or a two-parameter Fermi form [38]

$$
\rho_{A}(r)=\frac{\rho_{0}}{1+\exp \left(\frac{r-c}{a}\right)}
$$

for an exotic cluster $\left(A=A_{2}\right)$, heavier than the $\alpha$ particle, and the core nucleus with $\left(A=A_{1}\right)$. The parameters are taken as $c=1.07 A^{1 / 3}$ and $a$ is the diffuseness. $\rho_{0}$ is fixed by normalizing the total density to the mass number $A$.

For our purpose here we use also the Gaussian form factor of Ref. [30], herein referred to as (CEG83), whose functional 
TABLE V. Optimized parameters for SW3 potential from CEG83b.

\begin{tabular}{lcccc}
\hline \hline Cluster-core & $V_{0}(\mathrm{MeV})$ & $a(\mathrm{fm})$ & $x$ & $R(\mathrm{fm})$ \\
\hline${ }_{2}^{4} \mathrm{He}+{ }_{82}^{208} \mathrm{~Pb}$ & 191.000 & 0.740 & 0.320 & 7.112 \\
${ }_{4}^{10} \mathrm{Be}+{ }_{82}^{208} \mathrm{~Pb}$ & 465.510 & 0.918 & 0.410 & 7.297 \\
${ }_{6}^{14} \mathrm{C}+{ }_{82}^{208} \mathrm{~Pb}$ & 652.000 & 0.942 & 0.360 & 7.382 \\
${ }_{8}^{20} \mathrm{O}+{ }_{82}^{208} \mathrm{~Pb}$ & 923.600 & 0.956 & 0.320 & 7.502 \\
\hline \hline
\end{tabular}

form for the direct and the finite-range exchange components are given by

$$
\begin{aligned}
V_{D}(s) & =\sum_{i=1}^{3} G_{D_{i}}\left[\exp \left(-r_{v_{i}} s^{2}\right)\right], \\
V_{\mathrm{EX}}(s) & =\sum_{i=1}^{3} G_{E_{i}}\left[\exp \left(-r_{v_{i}} s^{2}\right)\right],
\end{aligned}
$$

with coefficients $G_{D_{i}}(\mathrm{MeV}), G_{E_{i}}(\mathrm{MeV})$, and $r_{v_{i}}\left(\mathrm{fm}^{-2}\right)$ given in Table I. We note that the strength of this latter effective interaction is density dependent through the Fermi momentum $k_{f}$. For the values displayed in Table I the maximum value $k_{f}=1.4 \mathrm{fm}^{-1}$ is used [30].

\section{RESULTS AND DISCUSSION}

Figure 1 compares the phenomenological (SW3) potential whose parameters are taken from Refs. $[12,16]$ with the microscopic interactions generated using the M3Y and CEG83 effective interactions. The microscopic interactions supplemented with both the zero-range (as in earlier studies $[17,28,29])$ and the finite-range exchange components are calculated using the modified computer code DFM [39].

The M3Y and CEG83a with the zero-range exchange interaction are seen to present similar shape characters. While their surface features are the same, the M3Y is deeper in the interior. The difference in their depths, which may be attributed to the density dependence of the CEG83a, increases with increasing cluster size from $\sim 10$ to $\sim 75 \mathrm{MeV}$. The CEG83b, with finite-range exchange interaction, has a shallower depth and seems to agree with or straddle the SW3 interaction. The large difference in the depths of the CEG83b compared to corresponding depths of the M3Y and/or the CEG83a, all un-normalized, shows that CEG83b more properly accounts for the medium effects. This view is reflected in the calcu-

TABLE VI. Experimental and calculated spectra of ${ }_{84}^{212} \mathrm{Po}$ in $\mathrm{MeV}$.

\begin{tabular}{lcccc}
\hline \hline $1^{\pi}$ & $E_{\text {expt }}$ & $E_{\mathrm{M} 3 \mathrm{Y}}$ & $E_{\mathrm{CEG} 83 \mathrm{a}}$ & $E_{\mathrm{CEG} 83 \mathrm{~b}}$ \\
\hline $0^{+}$ & 0.000 & 0.000 & 0.000 & 0.005 \\
$2^{+}$ & 0.727 & 0.585 & 0.586 & 0.600 \\
$4^{+}$ & 1.132 & 0.919 & 0.927 & 0.892 \\
$6^{+}$ & 1.355 & 1.336 & 1.367 & 1.268 \\
$8^{+}$ & 1.476 & 1.816 & 1.859 & 1.701 \\
$10^{+}$ & 1.834 & 2.297 & 2.361 & 2.142 \\
$12^{+}$ & 2.702 & 2.729 & 2.803 & 2.553 \\
$14^{+}$ & 2.885 & 3.044 & 3.123 & 2.878 \\
$16^{+}$ & & 3.152 & 3.210 & 3.057 \\
$18^{+}$ & 2.922 & 2.921 & 2.926 & 2.991 \\
$\mathcal{S}^{2}$ & & 0.42167 & 0.553 & 0.254 \\
\hline \hline
\end{tabular}

TABLE VII. Electromagnetic transitions calculated for different hybrid potentials along with experimental values in Weisskopf units (W.u.) for ${ }^{212} \mathrm{Po}$.

\begin{tabular}{lcccc}
\hline \hline Transitions & Expt. & M3Y & CEG83a & CEG83b \\
\hline $2^{+} \longrightarrow 0^{+}$ & $2.6 \pm 0.3$ & 2.8 & 2.8 & 3.2 \\
$4^{+} \longrightarrow 2^{+}$ & & 3.6 & 3.8 & 4.3 \\
$6^{+} \longrightarrow 4^{+}$ & $3.9 \pm 1.0$ & 3.9 & 3.9 & 4.5 \\
$8^{+} \longrightarrow 6^{+}$ & $2.3 \pm 1.0$ & 3.6 & 3.7 & 4.2 \\
$10^{+} \longrightarrow 8^{+}$ & $2.2 \pm 0.6$ & 3.2 & 3.3 & 3.7 \\
$12^{+} \longrightarrow 10^{+}$ & & 2.6 & 2.7 & 3.1 \\
\hline \hline
\end{tabular}

lated normalization constants, listed in Table II, for different core-cluster systems. Overall, the potential depth for all the interactions increases with cluster size. This is proportionate especially for the SW3 (and CEG83b) in agreement with earlier works. However, the microscopic nuclear potentials present flat or slightly rounded shapes in the internal region, while their surface parts are more diffusedthan the SW3. We exploit these properties together with their asymptotic character to construct the SW3 hybrid potential interaction corresponding to each of the effective interactions for the core-cluster systems.

Following Ref. [17] we stepped through the values of the mixing parameter $x$ and fitted the remaining parameters to the microscopic potentials (with $\lambda=1$ ) discretized in steps of $0.0004 \mathrm{fm}$. The fitting is achieved using the MATHEMATICA package for nonlinear fits. This is then followed by a proper renormalization of the depth $V_{0}$ using Eq. (6) in order to include the medium effects. To this end, the estimated energies of the ground-state band are found such that the quantity

$$
\mathcal{S}^{2}=\sum_{l}\left(E_{l}^{\mathrm{cal}}-E_{l}^{\mathrm{expt}}\right)^{2}
$$

is minimized. Tables III-V list the optimized parameters for the hybrid potential resulting from the different doublefolding effective interactions. The values generated using the M3Y (Table III) are seen to agree fairly well with those of Refs. $[17,29]$. The nuclear radii deduced from CEG83b in

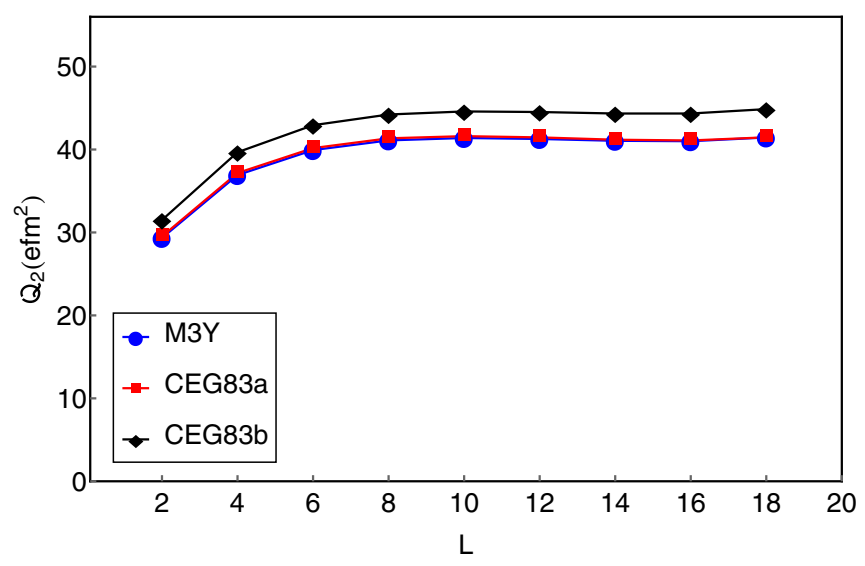

FIG. 2. Static quadrupole moments of $\alpha$ cluster states around ${ }^{208} \mathrm{~Pb}$ calculated for different potential model parameters. 
TABLE VIII. Experimental and calculated spectra of ${ }_{86}^{218} \mathrm{Rn}$ in $\mathrm{MeV}$.

\begin{tabular}{lcrrr}
\hline \hline $1^{\pi}$ & $E_{\text {expt }}$ & $E_{\mathrm{M} 3 \mathrm{Y}}$ & $E_{\text {CEG83a }}$ & $E_{\text {CEG83b }}$ \\
\hline $0^{+}$ & 0.000 & 0.000 & -0.006 & 0.000 \\
$2^{+}$ & 0.324 & 0.445 & 0.462 & 0.479 \\
$4^{+}$ & 0.653 & 0.624 & 0.636 & 0.647 \\
$6^{+}$ & 1.014 & 0.869 & 0.876 & 0.889 \\
$8^{+}$ & 1.393 & 1.172 & 1.181 & 1.178 \\
$10^{+}$ & 1.775 & 1.525 & 1.526 & 1.518 \\
$12^{+}$ & 2.169 & 1.919 & 1.920 & 1.902 \\
$14^{+}$ & 2.577 & 2.349 & 2.342 & 2.318 \\
$16^{+}$ & 3.002 & 2.805 & 2.797 & 2.765 \\
$18^{+}$ & 3.438 & 3.281 & 3.268 & 3.234 \\
$20^{+}$ & 3.859 & 3.768 & 3.750 & 3.716 \\
$22^{+}$ & 4.287 & 4.257 & 4.235 & 4.207 \\
$24^{+}$ & 4.725 & 4.737 & 4.710 & 4.693 \\
$26^{+}$ & 5.168 & 5.198 & 5.169 & 5.170 \\
$\mathcal{S}^{2}$ & & 0.335 & 0.348 & 0.415 \\
\hline \hline
\end{tabular}

Table V are larger than those obtained with M3Y and CEG83a with consequent reduction in the depth, mixing parameter, and diffuseness. We used these hybrid parameters to calculate the observables for each of the parent nuclei and compare the predictive ability of these DFM interactions.

We note here that the necessary correction to the corecluster potential in the region where the two densities overlap required to remove the underbinding of the $0^{+}[17]$ is achieved using

$$
V_{\delta}(r)=-\delta V * Q \text { for } r \leqslant r_{i}, \quad V_{\delta}(r)=0 \text { for } r \geqslant r_{i},
$$

where we search for the optimum values of $\delta V$ and $r_{i}$ over a two-dimensional mesh, and $Q$ is the decay energy.

\section{A. Spectroscopic analysis of ${ }^{212} \mathrm{Po}$}

The important feature of ${ }^{212}$ Po that makes it a good candidate for $\alpha$-clustering studies is that it exhibits proton and neutron pairs outside a doubly-closed shell giving the groundstate configuration $\left[\pi\left(h_{9 / 2}\right)^{2} v\left(g_{9 / 2}\right)^{2}\right]_{0^{+}}$. The level structures obtained, using Eq. (4), from the coupling of the $0^{+}$ground state of ${ }_{82}^{208} \mathrm{~Pb}$ to the core-cluster relative motion in Table VI is seen to agree fairly well with experimental data [41]. The position of the $0^{+}$state is obtained by taking the values $\delta \mathrm{V}=$ $3.800,3.924,4.450$, and $r_{i}=0.200 \mathrm{fm}$ for the coefficients in Eq. (30) for the M3Y, CEG83a, and CEG83b, respectively.

TABLE IX. Electromagnetic transitions calculated for different hybrid potentials along with experimental values in Weisskopf units (W.u.) for ${ }_{86}^{218} \mathrm{Rn}$.

\begin{tabular}{lcccc}
\hline \hline Transitions & Expt. & M3Y & CEG83a & CEG83b \\
\hline $2^{+} \longrightarrow 0^{+}$ & $>24.9$ & 27.2 & 27.5 & 30.3 \\
$4^{+} \longrightarrow 2^{+}$ & & 38.6 & 39.3 & 43.2 \\
$6^{+} \longrightarrow 4^{+}$ & & 42.1 & 42.8 & 48.1 \\
\hline \hline
\end{tabular}

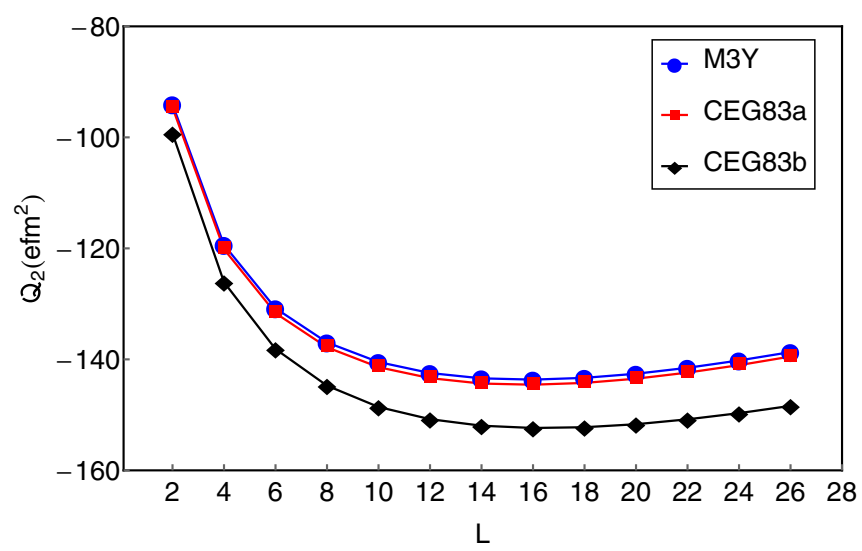

FIG. 3. Static quadrupole moments of ${ }^{10} \mathrm{Be}$ cluster states around ${ }^{208} \mathrm{~Pb}$ calculated for different potential model parameters.

We see that CEG83b gives better description probably due to its explicit account of the exchange term.

The calculated $B(E 2)$ transition strengths are compared with experimental data, taken from Ref. [40], in Table VII. The good agreement is obtained by introducing an effective charge $\varepsilon$ which reduces the $B(E 2)$ values from a factor of $\sim 2$ larger than experiment to the present level of agreement. We naively add to each fragment a charge correction term

$$
Z_{i} \equiv Z_{i}+\varepsilon A_{i}
$$

which represents a global way of including effects not explicitly taken into account in the model description. Since there is no universal choice, however, the effective charge is adjusted until the agreement between the estimated and measured values of a transition strength is achieved [23]. For ${ }^{212} \mathrm{Po}$ we used $\varepsilon=-0.886$ which reproduced the experimental value $B(E 2$ : $\left.6^{+} \longrightarrow 4^{+}\right)=3.9$ W.u. Although the agreement of our result with the recently measured $2^{+} \rightarrow 0^{+}$transition strength is good, the large negative value of the effective charge seems to support the conclusion of the authors of Ref. [40] on the $\alpha$ cluster structure of the $2^{+}$state of ${ }^{212} \mathrm{Po}$. We note again that CEG83b generates slightly enhanced values compared to other models indicative of a stretched wave function in the

TABLE X. Experimental and calculated spectra of ${ }_{88}^{222} \mathrm{Ra}$ in $\mathrm{MeV}$.

\begin{tabular}{lcccc}
\hline \hline $1^{\pi}$ & $E_{\text {expt }}$ & $E_{\mathrm{M} 3 \mathrm{Y}}$ & $E_{\mathrm{CEG} 83 \mathrm{a}}$ & $E_{\mathrm{CEG} 83 \mathrm{~b}}$ \\
\hline $0^{+}$ & 0.000 & 0.000 & 0.000 & 0.000 \\
$2^{+}$ & 0.111 & 0.102 & 0.091 & 0.109 \\
$4^{+}$ & 0.301 & 0.254 & 0.252 & 0.263 \\
$6^{+}$ & 0.55 & 0.468 & 0.468 & 0.481 \\
$8^{+}$ & 0.843 & 0.750 & 0.738 & 0.759 \\
$10^{+}$ & 1.173 & 1.078 & 1.073 & 1.091 \\
$12^{+}$ & 1.537 & 1.457 & 1.446 & 1.473 \\
$14^{+}$ & 1.933 & 1.879 & 1.871 & 1.901 \\
$16^{+}$ & 2.359 & 2.345 & 2.330 & 2.372 \\
$18^{+}$ & 2.811 & 2.845 & 2.833 & 2.884 \\
$20^{+}$ & 3.288 & 3.379 & 3.366 & 3.432 \\
$\mathcal{S}^{2}$ & & 0.045 & 0.050 & 0.051 \\
\hline \hline
\end{tabular}




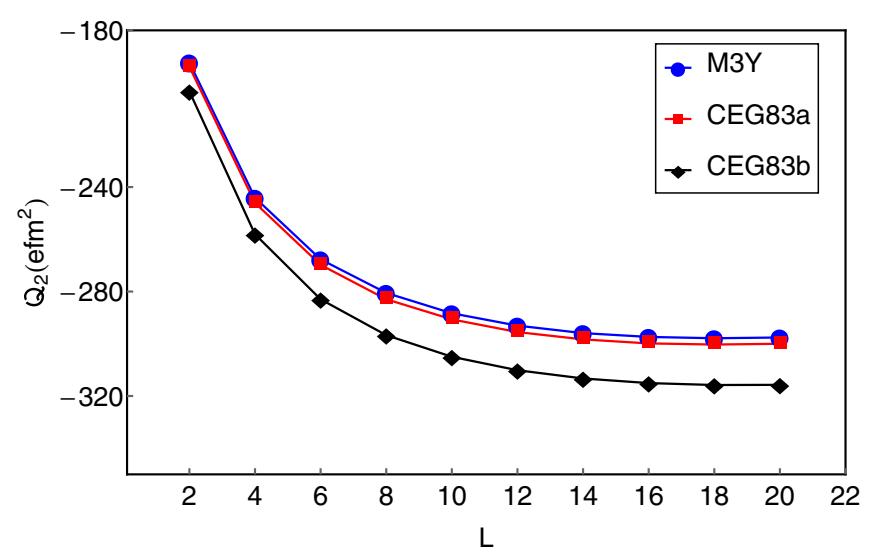

FIG. 4. Static quadrupole moments of ${ }^{14} \mathrm{C}$ cluster states around ${ }^{208} \mathrm{~Pb}$ calculated for different potential model parameters.

surface region. Figure 2 shows the predicted static moments plotted against angular momentum $l$ for the ground-state band. Again the model predictions of M3Y and CEG83a further show the similarity between the surface structures of the potential models. The values were obtained with correction added to the recoil term in Eq. (9), which yields positive values for the static moment. These positive values seem to indicate a prolate shape for the ground-state band of the parent nucleus corresponding to a very small deformation parameter of $\sim 0.012$. Our approximate value of the corresponding deformation parameter is thus in agreement with our basic assumption of a spherically symmetric system. It would therefore be interesting to compare the nearly constant value of the moments at $\approx 40 e \mathrm{fm}^{2}$ with experiment.

\section{B. Spectroscopic analysis of ${ }_{86}^{218} \mathrm{Rn}$}

Table VIII shows that the simple binary cluster model is able to reproduce almost absolutely the spectrum of the ground-state band of ${ }_{86}^{218} \mathrm{Rn}$ [41]. The different hybrid models have been supplemented with parameters $\delta V=$ $5.101,5.406,5.984$, and $r_{i}=0.100 \mathrm{fm}$. The $B\left(E 2: 2^{+} \longrightarrow\right.$ $0^{+}$) transitions in Table IX, extracted with no effective charge, are to be compared with the experimental value $B(E 2$ : $\left.2^{+} \longrightarrow 0^{+}\right)>24.9$ W.u. [41]. Figure 3 gives the quadrupole moments also calculated without the effective charge. In contrast with the results in Fig. 2 the values decrease with increasing angular momentum toward a constant value at high

TABLE XI. Electromagnetic transitions calculated for different hybrid potentials along with experimental values in Weisskopf units (W.u.) for ${ }_{88}^{222} \mathrm{Ra}$.

\begin{tabular}{lcccc}
\hline \hline Transitions & Expt. & M3Y & CEG83a & CEG83b \\
\hline $2^{+} \longrightarrow 0^{+}$ & 111 & 111.4 & 113.3 & 124.4 \\
$4^{+} \longrightarrow 2^{+}$ & 11.83 & 158.5 & 161.2 & 177.2 \\
$6^{+} \longrightarrow 4^{+}$ & & 173.3 & 176.1 & 193.7 \\
$8^{+} \longrightarrow 6^{+}$ & & 179.5 & 182.5 & 200.8 \\
$10^{+} \longrightarrow 8^{+}$ & & 181.9 & 184.9 & 203.7 \\
$12^{+} \longrightarrow 10^{+}$ & & 182.3 & 185.3 & 204.2 \\
\hline \hline
\end{tabular}

TABLE XII. Experimental and calculated spectra of ${ }_{90}^{228} \mathrm{Th}$ in $\mathrm{MeV}$.

\begin{tabular}{lcccc}
\hline \hline $1^{\pi}$ & $E_{\text {expt }}$ & $E_{\mathrm{M} 3 \mathrm{Y}}$ & $E_{\text {CEG83a }}$ & $E_{\text {CEG83b }}$ \\
\hline $0^{+}$ & 0.000 & 0.000 & 0.000 & 0.000 \\
$2^{+}$ & 0.058 & 0.068 & 0.065 & 0.068 \\
$4^{+}$ & 0.187 & 0.178 & 0.175 & 0.176 \\
$6^{+}$ & 0.378 & 0.342 & 0.338 & 0.337 \\
$8^{+}$ & 0.623 & 0.600 & 0.598 & 0.588 \\
$10^{+}$ & 0.912 & 0.870 & 0.863 & 0.858 \\
$12^{+}$ & 1.239 & 1.172 & 1.162 & 1.157 \\
$14^{+}$ & 1.600 & 1.556 & 1.544 & 1.539 \\
$16^{+}$ & 1.988 & 1.943 & 1.928 & 1.924 \\
$18^{+}$ & 2.400 & 2.398 & 2.379 & 2.378 \\
$20^{+}$ & 2.834 & 2.867 & 2.845 & 2.857 \\
$22^{+}$ & 3.283 & 3.383 & 3.358 & 3.365 \\
$\mathcal{S}^{2}$ & & 0.023 & 0.023 & 0.028 \\
\hline \hline
\end{tabular}

spin. The negative values seem to show the polarization of the spherical core tending toward the oblate deformation resulting from the motion of heavier clusters outside a closed core.

\section{Spectroscopic analysis of ${ }_{88}^{222} \mathrm{Ra}$}

It is well known that ${ }^{222} \mathrm{Ra}$ undergoes heavy ion $\left({ }^{14} \mathrm{C}\right)$ emission with decay half-life of $105.700 \mathrm{yr}$. Excellent agreement is obtained in Table X between the experimental [41], and calculated spectra from the three potential parameter sets where we have used $\delta V=0.624,0.300,0.700$, and $r_{i}=$ 0.05 . We see a decrease in the overlap region with the two fragments just touching each other and hence a decrease in the underbinding of the ground state $0^{+}$in comparison with results obtained with lighter clusters. The calculated value of the $B\left(E 2: 2^{+} \longrightarrow 0^{+}\right)$transition in Table XI agrees with the measured value taken from Ref. [18] given that $\varepsilon=0.106$ in Eq. (31). The value of the $B\left(E 2: 4^{+} \longrightarrow 2^{+}\right)$remains an unresolved mystery which in principle is expected to be higher with respect to $B\left(E 2: 2^{+} \longrightarrow 0^{+}\right)$. Figure 4 shows the static quadrupole moments of ${ }_{88}^{222} \mathrm{Ra}$ with oblate structure. The same remark concerning the tendency toward the shape holds as for the ${ }^{218} \mathrm{Rn}$ nucleus earlier discussed.

\section{Spectroscopic analysis of ${ }_{90}^{228} \mathrm{Th}$}

The experimental energy levels of ${ }^{228} \mathrm{Th}$ presented in Table XII is well reproduced by the model parameters cor-

TABLE XIII. Electromagnetic transitions calculated for different hybrid potentials along with experimental values in Weisskopf units (W.u.) for ${ }_{90}^{228} \mathrm{Th}$.

\begin{tabular}{lcccc}
\hline \hline Transitions & Expt. & M3Y & CEG83a & CEG83b \\
\hline $2^{+} \longrightarrow 0^{+}$ & $167 \pm 6$ & 168.8 & 171.7 & 187.2 \\
$4^{+} \longrightarrow 2^{+}$ & 242 & 240.9 & 244.9 & 267.1 \\
$6^{+} \longrightarrow 4^{+}$ & & 263.6 & 268.0 & 292.3 \\
$8^{+} \longrightarrow 6^{+}$ & & 272.9 & 277.5 & 302.9 \\
$10^{+} \longrightarrow 8^{+}$ & & 277.1 & 281.7 & 307.6 \\
$12^{+} \longrightarrow 10^{+}$ & & 279.3 & 283.9 & 310.2 \\
\hline \hline
\end{tabular}




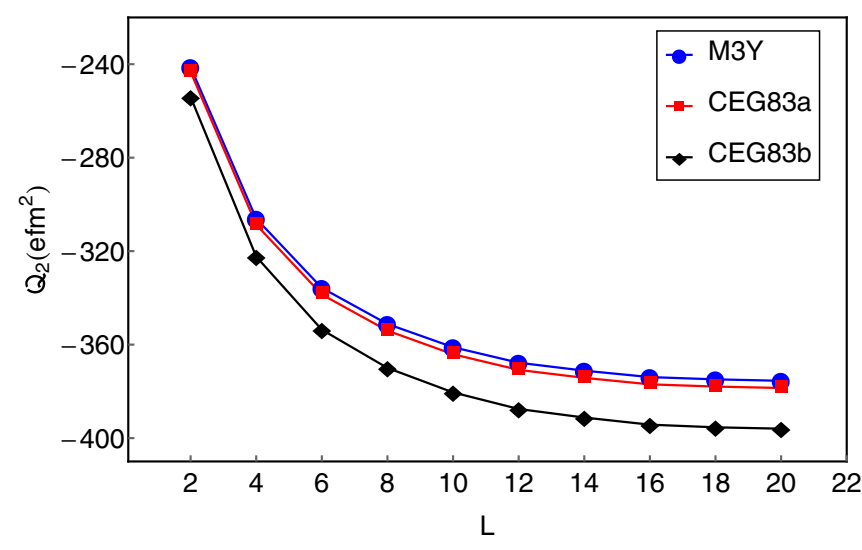

FIG. 5. Static quadrupole moments of ${ }^{20} \mathrm{O}$ cluster states around ${ }^{208} \mathrm{~Pb}$ calculated for different potential model parameters.

rected with $\delta V=1.200,1.200,1.500$, and $r_{i}=0.025$. In comparison with the results obtained for ${ }^{222} \mathrm{Ra}$, a similar remark can be made with regard to the underbinding nature of the $0^{+}$state. It is interesting to note that our model predictions for the $l^{\pi}=18^{+}$state agree with the assigned value of 2.400 $\mathrm{MeV}$ [41]. Similarly our model supports the $l^{\pi}=20^{+}$and $l^{\pi}=22^{+}$tentatively assigned to 2.834 and $3.283 \mathrm{MeV}$ levels [41]. The transition strengths in Table XIII, calculated with $\varepsilon=0.027$ taken for the effective charge, give results in good agreement with the experimental data. The near zero effective charge and of course the agreement may be due to the combined effect of the substantial increase in the cluster charge and the large amplitude of the wave function at the surface region. Comparing Fig. 5 with those discussed previously the increase in the absolute value of the static quadrupole moments with cluster size and charge is clearly observable.

\section{E. Decay lifetimes}

The estimated ground-state decay half-lives obtained with M3Y and CEG83a using Eq. (13) with preformation probability $P=1$ and the uncorrected decay energy of the ground state $Q=8.985 \mathrm{MeV}$ are approximately two times smaller than the measured values for ${ }_{84}^{212} \mathrm{Po}$ [41]. The CEG83b also yields a result with the same order of magnitude but which is about six times lower than the experimental value due possibly to the diffused surface of the potential. For ${ }_{86}^{218} \mathrm{Rn}$ our prediction for exotic decay half-life, using CEG83a with $P=$ 1 and the uncorrected $Q=14.360 \mathrm{MeV}$, is approximately of the same order as our previous results [17]. While a slightly

TABLE XIV. Decay half-lives obtained with different potential models.

\begin{tabular}{lcccc}
\hline \hline $\begin{array}{l}\text { Cluster-core } \\
T_{1 / 2}{ }_{2}^{4} \mathrm{He}+{ }_{82}^{208} \mathrm{~Pb} \\
(\mathrm{~ns})\end{array}$ & $\begin{array}{c}{ }_{4}^{10} \mathrm{Be}+{ }_{82}^{208} \mathrm{~Pb} \\
(\mathrm{~s})\end{array}$ & $\begin{array}{c}{ }_{6}^{14} \mathrm{C}+{ }_{82}^{208} \mathrm{~Pb} \\
(\mathrm{yr})\end{array}$ & $\begin{array}{c}{ }_{8}^{20} \mathrm{O}+{ }_{82}^{208} \mathrm{~Pb} \\
(\mathrm{yr})\end{array}$ \\
\hline Expt. & 300.000 & & $1.06 \times 10^{2}$ & $1.692 \times 10^{13}$ \\
M3Y & 158.000 & $6.345 \times 10^{18}$ & $5.40 \times 10^{3}$ & $1.389 \times 10^{14}$ \\
CEG83a & 142.466 & $2.680 \times 10^{18}$ & $3.60 \times 10^{3}$ & $7.815 \times 10^{13}$ \\
CEG83b & 52.214 & $4.207 \times 10^{17}$ & $4.27 \times 10^{2}$ & $5.663 \times 10^{12}$ \\
\hline \hline
\end{tabular}

TABLE XV. Mean-square charge radii and their experimental values in $\mathrm{fm}$.

\begin{tabular}{lcccc}
\hline \hline Nuclei & Expt. & M3Y & CEG83a & CEG83b \\
\hline${ }_{84}^{212} \mathrm{Po}$ & & 5.557 & 5.557 & 5.567 \\
${ }_{86}^{218} \mathrm{Rn}$ & 5.654 & 5.645 & 5.646 & 5.653 \\
${ }^{222} \mathrm{Ra}$ & 5.687 & 5.737 & 5.739 & 5.750 \\
${ }_{90}^{228} \mathrm{Th}$ & 5.749 & 5.854 & 5.857 & 5.870 \\
\hline \hline
\end{tabular}

larger result is obtained with M3Y, the value obtained with CEG83b is lower. The results are also found to be comparable with those of [42] upon the use of the preformation probability defined in our previous work [29]. For larger exotic clusters in ${ }_{88}^{222} \mathrm{Ra}$ and ${ }_{90}^{228} \mathrm{Th}$ with probability $P=1$ and $Q$ values corrected for electron shielding (i.e. $Q=33.153$ and $44.865 \mathrm{MeV}$, respectively), the calculated values grossly underestimate the experimental values by about 4-6 orders of magnitude. The improved results obtained with $P$ obtained in [29] are listed in Table XIV. We see that for the heavy clusters the CEG83b gives better predictions than M3Y and CEG83a.

\section{F. Mean-square charge radius}

The calculated rms charge radii for the $0^{+}$ground state of the parent nuclei are listed in Table XV. Except for ${ }^{212} \mathrm{Po}$ whose value is yet to be measured, the calculated rms radii agree with the experimental values in Ref. [43]. This is a remarkable test for the cluster wave function and hence the potential models.

\section{CONCLUSIONS}

In summary, we have constructed the hybrid potential model from different double-folding potential models to account for decay and spectroscopic nuclear properties of selected heavy nuclei within the binary cluster model. The ground-state band excitation energies are well reproduced, especially the low-spin states when the interior is corrected with a short-range interaction. The decrease in the degree of overlap from the light to exotic cluster, as indicated by the interaction range, seems to suggest a proper account of Pauli principle for the larger cluster mass. The enhanced transition probabilities and the static moments calculated with effective charge together with the rms radii for the ground-state band further confirm the consistency of our approach for most of the nuclei considered. We notice that for small cluster sizes satisfactory results are obtained for the ground-state decay half-lives. A proper account of preformation probabilities for both the $\alpha$ and exotic decays will expectedly resolve the remaining difference in the measured and calculated decay half-lives.

\section{ACKNOWLEDGMENTS}

B. D. C. Kimene Kaya thanks the National Institute for Theoretical Physics (NITheP) for financial support. This work was partly supported by South African National Research Foundation (NRF), Grand No. 807778. 
[1] K. P. Santhosh, R. K. Biju, and J. Antony, J. Phys. G: Nucl. Part. Phys. 35, 085102 (2008).

[2] A. I. Budaca, R. Budaca, and I. Silisteanu, Nucl. Phys. A 951, 60 (2016).

[3] A. Sandulescu and W. Greiner, J. Phys. G: Nucl. Phys. 3, L189 (1977).

[4] H. J. Rose and G. A. Jones, Nature 307, 245 (1984).

[5] S. Gales, E. Hourani, M. Hussonnois, J. P. Schapira, L. Stab, and M. Vergnes, Phys. Rev. Lett. 53, 759 (1984).

[6] P. B. Price, J. D. Stevenson, S. W. Barwick, and H. L. Ravn, Phys. Rev. Lett. 54, 297 (1985).

[7] S. W. Barwick, P. B. Price, and J. D. Stevenson, Phys. Rev. C 31, 1984 (1985)

[8] S. S. Malik and R. K. Gupta, Phys. Rev. C 39, 1992 (1989).

[9] G. Audi, O. Bersillon, J. Blachot, and A. H. Wapstra, Nucl. Phys. A 729, 3 (2003).

[10] W. Greiner, J. Y. Park, and W. Scheid, Nuclear Molecules (World Scientific, Singapore, 1995).

[11] B. Buck, A. C. Merchant, V. A. McBride, and S. M. Perez, J. Phys. G: Nucl. Part. Phys. 30, 1371 (2004).

[12] B. Buck, A. C. Merchant, and S. M. Perez, Phys. Rev. Lett. 65, 2975 (1990)

[13] H. Zheng, A. Bonasera, M. Huang, and S. Zhang, Phys. Lett. B 779, 460 (2018).

[14] M. Kimura, T. Suhara, and Y. Kanada-En'yo, Eur. Phys. J. A 52, 373 (2016).

[15] S. Saito, Prog. Theor. Phys. 62, 11 (1977).

[16] B. Buck, A. C. Merchant, and S. M. Perez, Phys. Rev. Lett. 76, 380 (1996)

[17] T. T. Ibrahim, S. M. Wyngaardt, and B. D. C. Kimene Kaya, Nucl. Phys. A 966, 73 (2017).

[18] E. J. du Toit, Masters thesis, University of Stellenbosch, 2014.

[19] B. Buck, A. C. Merchant, and S. M. Perez, Few-Body Syst. 29, 53 (2000).

[20] J. Cseh and W. Scheid, J. Phys. G. 18, 1419 (1992).

[21] T. M. Shneidman, G. G. Adamian, N. V. Antoneko, S. P. Ivanova, and W. Scheid, Nucl. Phys. A 671, 119 (2000).

[22] B. Buck, A. C. Merchant, S. M. Perez, and P. Tripe, Phys. Rev. C. 47, 1307 (1993).
[23] B. Buck and A. A. Pilt, Nucl. Phys. A 280, 133 (1977).

[24] T. T. Ibrahim and S. M. Wyngaardt, Phys. Rev. C 84, 044330 (2011).

[25] A. Soylu and S. Evlice, Nucl. Phys. A 936, 59 (2015).

[26] D. N. Basu, Phys. Lett. B 566, 90 (2003).

[27] D. N. Basu, J. Phys. G: Nucl. Part. Phys. 29, 2079 (2003).

[28] T. T. Ibrahim, S. M. Perez, and S. M. Wyngaardt, Phys. Rev. C 82, 034302 (2010).

[29] T. T. Ibrahim, S. M. Perez, S. M. Wyngaardt, B. Buck, and A. C. Merchant, Phys. Rev. C 85, 044313 (2012).

[30] N. Yamaguchi, S. Nagata, and T. Matsuda, Progr. Theor. Phys. 70, 459 (1983).

[31] Y. Sakuragi, Prog. Theor. Exp. Phys 2016, 06A106 (2016)

[32] D. T. Khoa, A. Faessler, and N. Ohtsuka, J. Phys. G: Nucl. Part. Phys. 16, 1253 (1990).

[33] D. N. Poenaru and W. Greiner, Phys. Scr. 44, 427 (1991).

[34] G. Andrzej and P. Krzystof, Nucl. Phys. A 451, 1 (1986).

[35] D. N. Poenaru, R. A. Gherghescu, and Walter Greiner, J. Phys. G: Nucl. Part. Phys. 40, 105105 (2013).

[36] P. Gulshani, Nucl. Phys. A 832, 18 (2010).

[37] G. R. Satchler and W. G. Love, Phys. Rev. 55, 183 (1979).

[38] B. Hahn, D. G. Ravenhall, and R. Hofstadter, Phys. Rev. 101, 1131 (1956).

[39] luky.jinr.ru/dfmpot

[40] D. Kocheva, G. Rainovski, J. Jolie, N. Pietralla, A. Blazhev, R. Altenkirch, S. Ansari, A. Astier, M. Bast, M. Beckers, T. Braunroth, M. Cappellazzo, A. Dewald, F. Diel, M. Djongolov, C. Fransen, K. Gladnishki, A. Goldkuhle, A. Hennig, V. Karayonchev, J. M. Keatings, E. Kluge, T. Kroll, J. Litzinger, K. Moschner, C. Müller-Gatermann, P. Petkov, M. Scheck, P. Scholz, T. Schmidt, P. Spagnoletti, C. Stahl, R. Stegmann, A. Stolz, A. Vogt, N. Warr, V. Werner, D. Wölk, J. C. Zamora, K. O. Zell, V. Y. Ponomarev, and P. VanIsacker, Phys. Rev. C 96, 044305 (2017).

[41] www.nndc.bnl.gov

[42] Z. Ren, C. Xu, and Z. Wang, Phys. Rev. C 70, 034304 (2004).

[43] I. Angeli and K. P. Marinova, At. Data Nucl. Data Tables 99, 69 (2013). 